Submitted to AJ

\title{
A Large Stellar Evolution Database for Population Synthesis Studies. III. Inclusion of the full Asymptotic Giant Branch phase and Web tools for stellar population analyses.
}

\author{
Daniel Cordier \\ Ecole Nationale Superieure de Chimie de Rennes, Campus de Beaulieu, F-35700 Rennes \\ daniel.cordier@ensc-rennes.fr \\ Adriano Pietrinferni ${ }^{1}$ \\ INAF - Astronomical Observatory of Collurania, via M. Maggini, I-64100 Teramo, Italy \\ pietrinferni@oa-teramo.inaf .it \\ Santi Cassisi ${ }^{2}$ \\ INAF - Astronomical Observatory of Collurania, via M. Maggini, I-64100 Teramo, Italy \\ cassisi@oa-teramo.inaf.it \\ and \\ Maurizio Salaris ${ }^{3}$ \\ Astrophysics Research Institute, Liverpool John Moores University, Twelve Quays House, \\ Birkenhead, CH41 1LD, UK \\ ms@astro.livjm.ac.uk
}

\begin{abstract}
${ }^{1}$ Universitá di Teramo, viale F. Crucioli I-64100, Teramo, Italy

${ }^{2}$ Instituto de Astrofisica de Canarias, Calle Via Lactea, E38205, La Laguna (Tenerife), Spain

${ }^{3}$ Max Planck Institut für Astrophysik, Karl-Schwarzschild-Strasse 1, Garching, D-85741
\end{abstract}


Stellar evolution tracks and isochrones are key inputs for a wide range of astrophysical studies; in particular, they are essential to the interpretation of photometric and spectroscopic observations of resolved and unresolved stellar populations. We have made available to the astrophysical community a large, homogenous database of up-to-date stellar tracks and isochrones, and a set of programs useful in population synthesis studies. In this paper we first summarize the main properties of our stellar model database (BaSTI) already introduced in Pietrinferni et al. (2004) and Pietrinferni et al. (2006). We then discuss an important update of the database, i.e., the extension of all stellar models and isochrones until the end of the thermal pulses along the Asymptotic Giant Branch. This extension of the library is particularly relevant for stellar population analyses in the near-infrared, or longer wavelengths, where the contribution to the integrated photometric properties by cool and bright Asymptotic Giant Branch stars is significant. A few comparisons with empirical data are also presentend and briefly discussed. We then present three web-tools that allow an interactive access to the database, and make possible to compute user-specified evolutionary tracks, isochrones, stellar luminosity functions, plus synthetic ColorMagnitude-Diagrams and integrated magnitudes for arbitrary Star Formation Histories. All these web tools are available at the BaSTI database official site: http://www.oa-teramo.inaf .it/BASTI.

Subject headings: astronomical data bases: miscellaneous - galaxies: stellar content - stars: general - stars: AGB and post-AGB

\section{Introduction}

The interpretation of photometric and spectroscopic observations of resolved and unresolved stellar populations is nowadays a fundamental tool to investigate the formation and evolution of galaxies. Large grids of stellar evolution models and isochrones are a necessary ingredient in this kind of analyses, together with appropriate tools to predict synthetic Color-Magnitude-Diagrams (CMDs) - hence star counts along the various observed CMD branches - integrated magnitudes and spectra of stellar populations with an arbitrary Star

Formation History (SFH). To this purpose Pietrinferni et al. (2004) and Pietrinferni et al. (2006) have published a huge and homogeneous database of stellar evolution models, that covers the relevant chemical composition range of stellar populations in galaxies of various morphological types, and allows one to choose among different treatments of core convection and stellar mass loss employed in the model calculations. These models are available on the 
web as downloadable files, from the BaSTI database - an acronym for "a Bag of Stellar Tracks and Isochrones" - at http://www.oa-teramo.inaf.it/BASTI, together with three web-tools that allow user-friendly manipulations of the stellar evolution library. Thanks to these tools one can compute user-specified isochrones, interpolate among evolutionary tracks to obtain the evolution for a mass value not contained in the grid, determine the differential and cumulative luminosity functions from a set of isochrones and, finally, compute synthetic stellar populations with an arbitrary SFH and determine their integrated magnitudes and colors.

This paper describes comprehensively the BaSTI database, and in particular how to run the various web-tools, the necessary inputs and their outputs. Next section describes the stellar evolution library, with particular emphasis on the most recent extensions not included in Pietrinferni et al. (2004) and Pietrinferni et al. (2006); the following three sections describe the individual web-tools that allow compute user specified isochrones and models, luminosity functions and synthetic CMDs. Final conclusions and an appendix with some more technical details about the implementation of the web-tools close the paper.

\section{Stellar evolution models and isochrones}

We have computed stellar evolution models for 11 different metallicities, namely $Z=$ $0.0001,0.0003,0.0006,0.001,0.002,0.004,0.008,0.01,0.0198,0.03$ and 0.04 , assuming two different heavy element distributions: the scaled-solar one by Grevesse \& Noels (1993), and the $\alpha$-enhanced one with $\langle[\alpha / F e]\rangle=0.40$ by Salaris \& Weiss (1998). As for the initial He-mass fractions, we employed a value $Y=0.245$ for the cosmological He (see, e.g., Cassisi et al. (2003)). To reproduce the solar initial He-abundance obtained from the calibration of the solar model, we assume an Helium enrichment law equal to $\Delta Y / \Delta Z \sim 1.4$. For each initial chemical composition we have computed stellar evolutionary tracks with mass in the range $0.5 \leq M / M_{\odot} \leq 10$ and a fine mass spacing. Mass loss from the stellar surface is accounted for using the Reimers (1975) law and two values of the free parameter $\eta$, namely $\eta=0.2$ and 0.4 . For stars that develop convective cores during the central H-burning phase we have computed models with and without overshoot from the Schwarzschild boundary of the central convective regions.

All evolutions, with the exception of the least massive stars whose central H-burning timescale is longer than the Hubble time, have been computed from the Pre-Main Sequence phase until to the C-ignition, or until the first thermal pulse along the Asymptotic Giant Branch (AGB), depending on the initial stellar mass. Full details of these calculations can be found in Pietrinferni et al. (2004) and Pietrinferni et al. (2006). Very recently we have 
extended the evolution of low- and intermediate-mass objects through the entire Thermal Pulse (TP) phase, until the end of the AGB stage. To this purpose we have used the synthetic AGB technique (Iben \& Truran (1978)). Our simplified treatment of the TP-AGB phase reproduces satisfactorily several integrated properties (see below) of stellar populations in the near-IR bands, which are greatly affected by the presence of TP stars. Given that the purpose of this library of stellar models is to provide a reliable tool to be employed in stellar population synthesis studies, we feel confident that the following simplified treatment of the AGB evolution is adequate for our purposes. For each stellar model of a given initial chemical composition and mass, we started the synthetic AGB evolution at the beginning of the TP phase, where the stellar evolution calculations were terminated. The first model of the synthetic TP-AGB evolution is characterized by the total mass $M$, Carbon-Oxygen core mass $M_{C O}$, luminosity $L$, effective temperature $T_{e f f}$ and surface chemical composition $\left(X_{f}, Y_{f}, Z_{f}\right)$ of the last fully evolutionary model.

The TP-AGB phase is then followed by increasing (after a given timestep $\mathrm{d} t$ ) $M_{C O}$ and $L$ according to Eqs. 5-7 in Wagenhuber \& Groenewegen (1998) which contain a term mimicking the effect of the Hot Bottom Burning, when appropriate. The hydrogen mass fraction in the envelope (an input of Eq. 6 of Wagenhuber \& Groenewegen (1998)) is approximated as $1-\left(Y_{f}+0.01\right)-Z_{f}$ all along the TP evolution. During a timestep the mass of the envelope $\left(M-M_{C O}\right)$ is reduced not only by the growth of $M_{C O}$, but also by mass loss processes, accounted for using the mass loss formulae by Vassiliadis \& Wood (1993). For any given value of $M$ and $M_{C O}$, the effective temperatures are computed using the relationships in Wagenhuber (1996), that are plotted in Fig. 8 of Wagenhuber \& Groenewegen (1998). To ensure continuity, the zero points of the equations describing the evolution of $L, M_{C O}$ and $T_{\text {eff }}$ have been adjusted to reproduce the corresponding values of the last fully evolutionary model, at the beginning of the TP phase. The synthetic evolution is stopped when the models have started to evolve off the AGB, at constant luminosity, towards their White Dwarf cooling sequence. The full models have been used to compute isochrones for ages ranging between $30 \mathrm{Myr}$ and 19 Gyr. We provide separately models and isochrones with and without the inclusion of the TP-AGB phase, in case users wish to use their own description of the TP-AGB evolution, either employing fully evolutionary calculations, or synthetic AGB models with different choices about, i.e., mass loss and/or core mass-luminosity relationship. To this purpose we provide - as mentioned below - tables with He and CO core mass as well as the luminosity and effective temperature at the beginning of the TP-AGB phase.

The reader is referred to Pietrinferni et al. (2004) for a detailed discussion about the normalization of the individual evolutionary tracks (the concept of key-point and normalized points between two successive key-points) and isochrone computation. Here we simply mention two changes with respect to the discussion in Pietrinferni et al. (2004). The first change 
is the inclusion of two additional key-points along the Red Giant Branch (RGB) one to mark the maximum luminosity during the RGB bump, and another one to mark the minimum RGB bump luminosity (key points 6 and 7). A total of 370 normalized points is distributed between key point 6 and its predecessor, 30 points are distributed between key-points 6 and 7, and 400 points are distributed between key-point 7 and the following one, the tip of the RGB. Due to the TP-AGB extension, we have added an additional key-point (number 17) after the beginning of the TP phase, to mark the termination of the AGB phase, when the model evolution turns to the blue. A total of 250 normalized points cover the TP-AGB phase, between the key-points 16 and 17. The resulting isochrones have a total of 2250 points.

The main characteristics of the stellar model database are summarized in Tables 1 and 2. Table 1 lists the grid of initial chemical compositions; Table 2 summarizes the available sets of models and isochrones. For a given heavy elements mixture (either scaled-solar or $\alpha$ enhanced) we have computed models for $11 Z$ values (Table 1 ) using both $\eta=0.2$ and $\eta=0.4$ for the evolution until the TP-AGB phase. For each $\eta$ we have computed models with and without overshoot from the central convective core (in the appropriate mass range). The extension of the overshooting region $\lambda_{O V}$ is $0.2 H_{p}\left(H_{p}\right.$ is the local pressure scale height at the Schwarzschild convective boundary) decreasing to zero when the convective cores vanish, as described in Pietrinferni et al. (2004). For each choice of the metal mixture, $Z, \eta$ and convective core extension we specify the number of tracks available, their mass range, the number of isochrones available and their age range. Notice that for masses above $\sim 2.4 M_{\odot}$ the evolution with $\eta=0.4$ is identical to the case of $\eta=0.2$ (because of the negligible amount of mass lost during their evolution, according to the Reimers' law).

Broadband magnitudes and colors of the stellar evolution tracks and isochrones are predicted using color- $T_{\text {eff }}$ transformations and bolometric corrections based on an updated set of model atmospheres described in Pietrinferni et al. (2004) and Cassisi et al. (2004). The evolutionary results are available in the photometric filters listed in Table 2. Additional filters will be added with time. It is worth to point out that, for the first time, we have homogenoeus transformations for both scaled-solar and $\alpha$-enhanced mixture even for supersolar metallicities. At present, the transformations for the ACS HST filters are available only for a scaled-solar chemical composition.

For the TP-AGB section of the models and isochrones broadband colors and magnitudes have been computed by supplementing the transformations used up to the beginning of the TP phase with the Westera et al. (2002) ones for RGB and AGB stars with $T_{\text {eff }}<3750 \mathrm{~K}$. To ensure continuity, the bolometric corrections and colors of Westera et al. (2002) have been shifted to match the other sets of transformations when $T_{\text {eff }}=3750 \mathrm{~K}$. Along the TP-AGB 
phase, when the $(J-K)$ colors reach $(J-K)=1.2 \mathrm{mag}$, we used the $(J-K)-T_{\text {eff }}$ and $(H-K)-T_{\text {eff }}$ relationships by Bergeat et al. (2001) that are appropriate in the carbon star regime. Models and isochrones extended along the TP-AGB phase are at the moment transformed only to the $U B V R I J H K L$ system.

All models described above have been computed in the assumption that a star of a given mass and initial chemical composition loses during its evolution a fixed amount of mass, determined by the adopted mass loss law. An isochrone for an old population will therefore display on the CMD a clumpy Horizontal Branch (HB) phase, made of stars all essentially with the same total mass, because of both the constant mass of their progenitor, and the constant value of the mass lost along the previous RGB phase. If one wants to simulate the extended HBs observed in Galactic globular clusters, a spread in the amount of mass lost by the RGB progenitor has to be accounted for. To this purpose, for each chemical composition we have also computed additional large sets of core He-burning models, with He core mass and envelope chemical profile fixed by a RGB progenitor having an age of $\sim 13$ Gyr at the RGB tip, and a range of values of the total stellar mass, to simulate the effect of a spread in the mass lost along the RGB. These Horizontal Branch (HB) models ( 30 for each chemical composition) constitute a valuable tool to perform synthetic HB modeling, and to investigate pulsational and evolutionary properties of different kinds of variable stars.

Users can directly download from the BaSTI database evolutionary tracks and HB models as single files, or as tar gzipped archive files, selecting among the following options (11 initial chemical compositions for each choice): scaled-solar, $\alpha$-enhanced, canonical (noovershoot), non-canonical (overshoot included), $\eta=0.2, \eta=0.4$, TP-AGB excluded, TP-AGB included. We have included in the web page appropriate readme files and provide also tables (both in ascii and html format) summarizing the main properties of the theoretical models (not including the TP-AGB phase) and providing also the CMD location of the Zero Age HB (ZAHB) and the central He exhaustion stages. These two latter sets of tables can be found at the pages containing the $\eta=0.4$ models. A large number of precomputed isochrones for each of the individual options described before - spanning the full age range allowed by the computed models - can be downloaded as tar gzipped archive files.

The models and isochrones contained in BaSTI have been extensively tested in Pietrinferni et al. (2004) and Pietrinferni et al. (2006), and already employed in a number of investigations (e.g., Gallart et al. (2005)). We conclude this section showing a few tests for the TP-AGB extension we introduced in this paper. We have first tested our isochrones against near-IR Surface-Brightness-Fluctuation (SBF) magnitudes, that are very sensitive to the brightness and evolutionary timescales of AGB stars (see, e.g., Liu et al. (2000), Cantiello et al. (2003), 
Mouhcine et al. (2005), Raimondo et al. (2005)).

We considered the $J$ and $K$-band SBF magnitudes determined by González et al. (2004) for a sample of Magellanic Clouds' superclusters. A supercluster is obtained by coadding individual clusters belonging to the same SWB class (see Searle et al. (1980) for the definition of the SWB classes, that are essentially age ranges). González et al. (2004) provide also the mean metallicity and age of the objects grouped in each supercluster. The original SBF magnitudes were on the 2MASS system and have been transformed to the Johnson system following Bessell \& Brett (1988) and Carpenter (2001).

Figures 1 1and 2 display the observed SBF absolute magnitudes in $K$ and $J$ (González et al. (2004) assume $(m-M)_{0}=18.50 \pm 0.13$ for the Large Magellanic Cloud (LMC) and $(m-M)_{0}=18.99 \pm 0.05$ for the SMC) of 6 superclusters - whose age range is covered by our models - as a function of age. Different symbols correspond to different mean metallicities; the three youngest superclusters share all the same metallicity. Horizontal error bars correspond to the age spread around the mean supercluster ages, as given by González et al. (2004).

We have compared these data with the SBF magnitudes obtained from our isochrones, for the appropriate mean metallicities of the individual superclusters. Notice that for each individual $Z$ considered, we have plotted the theoretical SBF magnitudes only in the age range spanned by the one (or more) supercluster with that mean value of $Z$. We employed the BaSTI models with $\eta=0.2$, including overshooting in the appropriate age range. For the lowest metallicity (oldest) supercluster we display also the results obtained with $\eta=0.4$. Theory is able to fit the data points within the observational errors, the only exception being the $J$-band SBF of the oldest supercluster.

Figure 3 compares the dereddened integrated $(J-K)$ and $(H-K)$ colors provided by González et al. (2004) for the 6 superclusters - transformed to the Johnson system as done for the SBF - with theoretical predictions from the same models employed for the SBF analysis. Theoretical colors appear consistent with the observational counterpart; the mean difference between theory and observations is equal to $\sim 0.03$ mag in both $(J-K)$ and $(H-K)$. This value can be considered a reasonable estimate of the accuracy of our predicted integrated near-IR colors. Finally, Fig. 4 compares the combined $K-(J-K)$ CMDs of AGB stars in a sample of LMC clusters, with theoretical isochrones. The individual stars have been coadded according to the SWB class of the parent clusters. Reddenings are from Frogel \& Cohen (1982) and we used an LMC distance modulus $(m-M)_{0}=18.50$. The metallicities and ages of the theoretical isochrones have been selected on the basis of the SWB class following again González et al. (2004). One can notice a good overlap between the theoretical AGB sequences and the observed near-IR CMDs. 


\section{Isochrone Maker and Track interpolation program}

We have realized a dedicated WEB interface that allows a direct computation of isochrones for any given choice of age, for the chemical compositions and within the age ranges listed in tables $\mathrm{A}$ and 2. It also enables the user to compute interpolated evolutionary tracks in the mass range $0.5-10 M_{\odot}$ for any chemical composition in the grid. A linear interpolation on a point-by-point basis between two neighbouring evolutionary tracks stored in the database is performed, to determine the track for the specified mass. The mass spacing of the tracks included in BaSTI is small enough that a linear interpolation is sufficient to guarantee a high accuracy. Once the track is computed, it is immediately transposed in the chosen photometric system.

The use of this interface is particularly simple: first of all one has to get to the server using this URL:

$$
\text { http://www.oa-teramo.inaf.it/BASTI } 1
$$

and click on the link Isochrones - Tracks in the Web Tools section; a page like the one displayed in Fig. 5 will appear. The user has now to choose among a number of options:

- the output type, i.e., isochrone or interpolated evolutionary track;

- the heavy elements mixture, i.e. scaled-solar or $\alpha$-enhanced;

- the photometric system of interest; at the moment we allow to choose between the standard $U B V R I J H K L$ filters and the ACS-HST ones;

- core convection treatment, i.e. with or without convective core overshoot;

- the initial chemical composition $(Y, Z)$.

Once these input parameters are fixed, the next screen allows the choice of the isochrone age (in Myr) or the track initial total mass in solar units, and the job submission. Outputs (in the same format of the tracks and isochrones stored in the database) are directly sent to the user's browser.

\footnotetext{
${ }^{1}$ In case of Network and/or technical problem with this official web site, the user can connect to the D. Cordier's personal site: http://astro.ensc-rennes.fr where the BaSTI web-tools have been mirrored in the BaSTI Web Tools section.
} 


\section{Luminosity Function Program}

The isochrones stored in the BaSTI database, or obtained with the web-tool described in the previous section, can be used as input for the tool described here, that computes the luminosity functions (e.g. differential and cumulative star-counts as a function of the magnitude in a given wavelength band) of single-age, single-metallicity stellar populations.

To run this web-tool the user has to provide 4 parameters: the number of isochrones whose luminosity functions are needed (maximum 20); the photometric filter (in the UBVRIJKLH system); the Initial Mass Function (IMF) exponent; the size of the magnitude bins within which the star counts are performed (e.g., $0.15 \mathrm{mag}$; these bins have to be larger than 0.05 mag). We adopt for the IMF a form of this type: $\frac{d N_{\star}}{d M}=C M^{-\alpha}$ where $d N_{\star}$ is the number of stars formed with mass between $M$ and $M+d M$, and $C$ is a normalization constant whose value is fixed by imposing that the total number of stars populating the isochrones is equal to $10^{6}$. A value of $\alpha$ equal to 2.35 corresponds to the Salpeter (1955) IMF.

One has then to choose between the options of computing the luminosity functions only until the RGB tip, along the whole isochrone until the Early-AGB, or including also the TP-AGB phase. Star counts are then computed analytically by convolving the IMF with the distribution of the initial stellar mass values along the isochrones. For example, the number of stars $N$ between two consecutive points $i-1$ and $i$ along a chosen isochrone is given by: $N=\frac{C}{1-\alpha}\left(M_{i}^{1-\alpha}-M_{i-1}^{1-\alpha}\right)$.

As a final step one has to upload the isochrone files, and submit the job. Results are directly sent to the user Internet browser as ASCII data, that are divided into several sections, each of them corresponding to one individual isochrones. The input parameters are recalled: metallicity, IMF exponent, age, etc. The data themselves are displayed in three columns: the mean magnitude of the bin, the differential $(\log N$ - where $N$ denotes the star counts per magnitude bin as a function of the magnitude) and cumulative ( $\log N+-$ where $N+$ denotes the sum of the star counts from the faintest bin to the actual one, as a function of their magnitude) luminosity functions.

\section{Synthetic CMD code}

The computation of synthetic CMDs is often required to interpret observations of resolved or unresolved stellar populations. The simplest form of a synthetic CMD is an isochrone, that represents the sequence occupied by stars all formed at the same time and with the same initial chemical composition. Clearly an isochrone does not directly contain information about the number of stars populating the various CMD branches, nor the effect 
of photometric errors and reddening, and cannot represent composite stellar populations made of multiple generations of stars. To include these effects one needs a synthetic CMD generator. In the BaSTI website the user can access the SYNTHETIC MAN(ager) code that is an evolution of the version briefly described in Pietrinferni et al. (2004). This code computes magnitudes and colors of objects belonging to a synthetic stellar population with an arbitrary Star Formation History (SFH). The program employs a grid of isochrones with ages between $30 \mathrm{Myr}$ and 14 Gyr (we employ 49 isochrones extended until the end of the TP-AGB phase, for each of the 11 metallicities included in our model grid).

The SYNTHETIC MAN code is based on a Monte-Carlo algorithm, that allows one to include in a simple way observation-related effects like reddening/distance/metallicity dispersion and photometric errors, and to identify on a star-by-star basis the various types of pulsating stars that may be present in the synthetic population. Synthetic CMDs and observation-related effects can also be efficiently determined by means of analytical integrations, as done in (Dolphin 2002). It is worth noticing that Skillman et al. (2003) applied three different methods to determine the SFH of the galaxy IC1613. One of them is the analytical technique by Dolphin (2002), another one is a Monte-Carlo method similar to the one we implemented. A comparison of the results with the different techniques shows striking agreement.

The general structure of the SYNTHETIC MAN code is as follows. An SFH file has to be specified first. The SFH contains a grid of $N$ ages $t_{i}$ (the upper limit for $N$ is set to 200) increasing with increasing running index $i$ (the present time is assigned $t=0$ ) and for each age one has to specify a scale factor $S F_{i}$ proportional to the relative number of stars formed at that time. Also, the metallicity (parametrized by $[\mathrm{Fe} / \mathrm{H}]$ ) of the stars formed at that age has to be given $\left([\mathrm{Fe} / \mathrm{H}]_{i}\right)$ together with a $1 \sigma$ Gaussian spread around this value. The scale factor and $[\mathrm{Fe} / \mathrm{H}]$ for ages between $t_{i} \leq t<t_{(i+1)}$ are then assumed to be equal to $S F_{i}$ and $[\mathrm{Fe} / \mathrm{H}]_{i}$, respectively. In other words, the $\mathrm{SFH}$ is considered to to be a sequence of step functions. The scale factor and $[\mathrm{Fe} / \mathrm{H}]$ at $i=N$ are not considered and can be set to any arbitrary value; the last point in the SFH file is important only because it provides the upper age-limit for the simulated stellar population. If one or more single burst stellar populations are needed, one or more pairs of identical ages (i.e. $t_{i}=t_{(i+1)}$ ) need to be included in the SFH file.

After the SFH is read by the code, the following cycle starts, running along the SFH index, from $i=0$ to $i=N$. The age difference between two generic $t_{i}$ and $t_{(i+1)}$ is computed, and for each star formed in this time interval (the number of stars formed in a generic time interval is determined from the input SFH) a random value of the stellar age $t_{i} \leq t<t_{(i+1)}$ is drawn with a flat probability distribution, together with a mass $M$ selected randomly 
according to a user specified IMF (the default mass range goes from $0.1 M_{\odot}$ to $120 M_{\odot}$, although a different lower mass limit can be specified by the user) and a random value of $[\mathrm{Fe} / \mathrm{H}]$ assigned according to the value of $[\mathrm{Fe} / \mathrm{H}]_{i}$ plus the $1 \sigma$ spread given in the SFH. With the three specified values of $t, M$ and $[\mathrm{Fe} / \mathrm{H}]$ the program interpolates quadratically in age, metallicity and then mass among the isochrones in the grid, to determine the star luminosity, effective temperature, actual value of the mass (in principle different from the initial value because of mass loss) and photometric properties in the UBVRIJHKL system.

For each generated mass an additional random number determines whether the star is member of an unresolved binary system (the percentage of unresolved binaries has to be specified as input); if this is true, the mass of the second component is selected randomly following Woo et al. (2003) and the fluxes of the two unresolved components are properly added.

Once the photometric properties of an object (single or belonging to an unresolved binary system) are determined, depth effects of the synthetic stellar population are simulated probabilistically according to a uniform stellar distribution with a user specified total depth (in mag). After depth effects are included, the individual magnitudes are modified by adding the effect of extinction (another input parameter; we have employed the extinction ratios from the Asiago Database on Photometric Systems - see Moro \& Munari (2000) - and $R_{V}=3.1$ ) and then further perturbed according to a Gaussian distribution, to simulate the photometric errors, with a user specified $1 \sigma$ width. The star $[\mathrm{Fe} / \mathrm{H}]$ value is also perturbed according to a Gaussian error with user specified $1 \sigma$ width, to mimic the effect of spectroscopic observational errors. As an additional option, the code can search for variable stars, according to their location with respect to the boundary of RR Lyrae and Cepheid instability strips; pulsation periods are then determined. Table 3 shows literature references for the relevant type of variables.

Once all stars formed between ages $t_{i}$ and $t_{(i+1)}$ are generated, the next interval $t_{(i+1)} \leq$ $\left.t<t_{(i+2)}\right)$ is considered, and the cicle continues, ending when all stars in the final age bin $t_{(N-1)} \leq t<t_{(N)}$ are generated.

As a technical comment, if the value of $M$ for a star generated by the Monte-Carlo procedure is too large to be still evolving at its age $t$, or is lower than $0.5 M_{\odot}$, the contribution to the total mass of stars formed in the population is taken into account, but the star photometric properties are not determined. The total mass of the objects formed, the total number of the stars whose photometric properties have been determined (in principle different from the total number generated for the reasons mentioned above) plus integrated magnitudes (in the $U B V R I J H K L$ system) of the synthetic population are also computed. 
Like all Monte-Carlo based simulations, our program makes an extensive use of random numbers. Our random number generator (initially written by James (1990)) needs seeds to be initialized; these seeds can be either provided by the user or got from http://www.random.org which derived true random numbers from atmospheric electromagnetic noise.

The web interface to SYNTHETIC MAN can been found in the BaSTI section called: Synthetic Color - Magnitude Diagrams. This software is as simple to use as the others but a registration is required before its first use. The user receives a notification by e-mail when the computations are completed. In order to get an user identification, one needs to contact S. Cassis: $2^{2}$ or D. Cordier ${ }^{3}$. In the following we summarize the input parameters for a run of SYNTHETIC MAN.

- A scale factor for the SFH. As explained before, the SFH contains the relative weight of the star formation episodes in the various age bins. In order to fix the absolute number of objects in each stellar generation, a scale factor has to be chosen. The product of this scale factor times the relative weight in the input SFH gives the total number of stars created in a generic age bin, with mass between 0.1 (or a user specified lower mass limit) and $120 M_{\odot}$. If more than $210^{6}$ objects are expected to be formed at a given time step, the program will stop to highlight the excessive amount of computational time needed for the whole simulation.

- A choice for the mean photometric error. The user can adopt a $1 \sigma$ constant error that it is applied to all stars in the simulation, in all 9 photometric bands. The size of this constant error (between 0.0 and $1.0 \mathrm{mag}$ ) has to be specified. Alternatively, the user can choice to adopt an error varying with the actual star magnitude and/or photometric band. The exact values have to be specified by the user in an appropriate input file. This photometric error input file contains 18 columns and an arbitrary number $N$ of rows (upper limit $N=200$ ). The first 9 columns display $N$ magnitudes $M_{\lambda}^{i}$ ( $i$ running from 1 to $N$ ) in order of increasing values, for the $U B V R I J H K L$ photometric bands respectively. The remaining 9 rows are the corresponding $1 \sigma$ photometric errors in the $U B V R I J H K L$ filters, respectively (e.g., $\sigma_{U}, \sigma_{B}, \sigma_{V}$ and so on). The choice of the error for any individual synthetic star proceeds as follows. If $M_{V}^{\star}$ is the star magnitude in the $V$ band, the program searches for a pair of neighboring tabulated $M_{V}^{i}$ magnitudes such that $M_{V}^{i}<M_{V}^{\star} \leq M_{V}^{i+1}$, and assigns to the star $\sigma_{V}$ the value tabulated for $M_{V}^{i+1}$.

\footnotetext{
${ }^{2}$ cassisi@oa-teramo.inaf.it

${ }^{3}$ daniel.cordier@ensc-rennes.fr
} 
If $M_{V}^{\star}$ is smaller (larger) than $M_{V}^{1}\left(M_{V}^{N}\right)$ the error corresponding to $M_{V}^{1}\left(M_{V}^{N}\right)$ is used. The same procedure is repeated for all other photometric bands considered.

- The $1 \sigma$ mean spectroscopic error (between 0.01 and 1.0 dex).

- A choice for range considered in the stellar mass random extraction. An upper limit of $120 M_{\odot}$ is always considered. The user can decide to use a different lower mass limit (larger than $0.1 M_{\odot}$ ) that has then to be specified by the user.

- The total spatial depth of the population (between 0.0 and $10.0 \mathrm{mag}$ ).

- The colour excess $E(B-V)$ (between 0.0 and $10.0 \mathrm{mag})$.

- The fraction of unresolved binaries.

- The minimum mass ratio for the unresolved binary systems, that enters the Woo et al. (2003) relationship (between 0.0 and 1.0)

- The IMF. The user can adopt a power law of the form $M^{-x}$ is employed. The exponent $x$ has then to be specified. Alternatively, the user is allowed to choice the Kroupa et al. (1993) IMF.

- Choice of the isochrone set to use according to the value of an integer index between 1 and 8. The correspondence between integer value and isochrone set is reported in Table 4

- Possibility to search for pulsating variable stars harbored by the synthetic population, and compute their periods. An integer value equal to 1 enables the search, a value equal to 0 prevents the search.

- Input the desired SFH. A number of pre-specified SFHs for selected galaxies are also provided (see Table 50).

- As all simulations based on a Monte-Carlo method, our program needs seeds for random numbers generator initialization. The user can choose the values of the seeds, or let the program automatically get seeds from the server of entropy http://www.random.org. We underline that all computations initialized with the same seeds will lead to identical outputs.

After specifying the input parameters, users can start their computations by clicking the "Submit" button. The calculation may take some time (from a few minutes to hours, mainly depending on the requested number of stars). After the calculation is completed the 
user receives an e-mail as notification. As the size of the output files can amount to several MBytes, they cannot be sent by e-mail and a solution involving a dynamically generated web page has been found preferable. In the output web-page four data files are available:

- Synth_Pop_*_user.in: this ASCII file recalls the parameters used for the calculation.

- Synth_Pop_*_.HRD.gz: this gzipped file contains data for the individual stars, in which one can find the following quantities: identification number, $\log (t)(\mathrm{yr}),[\mathrm{Fe} / \mathrm{H}]$, the value of the actual mass, $\log \left(\mathrm{L} / \mathrm{L}_{\odot}\right), \log \left(\mathrm{T}_{\text {eff }}\right), M_{V},(B-V),(U-B),(V-I),(V-R)$, $(V-J),(V-K),(V-L),(H-K)$, the value of the initial mass, the initial mass of the unresolved binary companion (if present - for stars without companion this quantity is set to 0.0), logarithm of the pulsation period (in days - if the search for variable is off or if the star is not pulsating this quantity is set to 99.99), an index denoting the type of variable (see Tab. 3 for explanations). This file can be uncompressed with the standard GNU software gunzip. As this file can be relatively large, the downloading is automatically forced when users click on the link.

- Synth_Pop_*_.sfh: contains the Star Formation History data used for the calculations.

- Synth_Pop_*_. INT_PROPERTIES: file with the integrated UBV RIJHKL absolute magnitudes produced by the stars evolving in the synthetic population, several selected integrated colors, the total number of stars with computed photometric properties, the total mass of stars formed (within the mass range specified in the input file) according to the specified SFH and a summary of the various types of variable stars found (if the search for variables is off the values are all equal to zero).

The symbol * denotes a string chosen by the WEB tool, to avoid that output files corresponding to a specific simulation are overwritten by those related to a different run. The string is selected according to the time when the numerical run has been launched. For example, if the run is launched at 16.51.34 of May the 3rd of the year 2006, the string will be 'May_3_16.51.34_2006'

An example of the output web-page is displayed in Fig. 6. The $V-(B-V)$ Hess's diagram taking into account all the synthetic objects drawn for the simulation is also automatically displayed, stars numbers being encoded with colors.

The user should be aware that - for obvious reasons - web pages displaying program outputs are deleted by the software manager one month after the end of the computation. 


\section{Conclusion}

In this paper we have presented the content of the BaSTI database for stellar population synthesis studies. In particular, we have discussed the recent extension of the models until the end of the TP-AGB phase, and described three web-tools to compute synthetic CMDs, luminosity functions and user-specified isochrones and stellar evolution tracks.

In the near future the BaSTI database will be developed following two directions: the extension of the database itself and the development of an on-line based version of our stellar evolution code.

We plan to extend the mass range spanned by our database, by adding tracks for very low mass objects (below $0.5 M_{\odot}$ ) and very massive stars (above $10 M_{\odot}$ ), the latter until Cburning ignition.

With a Web-based version of our evolution code, people who are not expert in stellar evolution calculations will be able to compute models with their customized set of parameters (mixing length value, extension of the overshooting region, metallicity, etc), avoiding inaccurate interpolation and/or extrapolation between and/or from existing models. That could also allow for more complex tuning like: opacity tables or nuclear rate table switching, change of equation of state, etc. We think that this Web-based evolutionary code, as well as the $B a S T I$ database could be relevant tools for the whole scientific community within the ongoing Virtual Observatory" project (Pasian 2004).

We wish to thank an anonymous referee for suggestions that helped us to improve the content and readability of the manuscript. D. Cordier thanks the Ecole Nationale Supérieure de Chimie de Rennes for working facilities and M.J. Goupil and Y. Lebreton for so many things. We wish also to acknowledge all people who have already used and/or will be using the BaSTI web-tools for their own research, and who will send us their comments/suggestions. We warmly thank E. Sandquist for all the useful comments and for pointing out the existence of some problems in a preliminary version of our WEB tools. S.C. warmly thanks financial support from MIUR and INAF.

\section{A. BaSTI Web Tools: some technical aspects}

The World Wide Web (WWW), originally conceived for document delivery, has evolved into a medium supporting interactive computations. One way for a web server (in our case APACHE, see http://www . apache.org) to interact with data-generating programs is the well known Common Gateway Interface (hereafter CGI). Input parameters and data provided by 
the user are passed to our three FORTRAN programs through PERL programs. PERL is probably the most common CGI scripting language, for which an huge collection of modules is available in the Comprehensive Perl Archive Network (see http://www. cpan.org).

The tracks/isochrones interpolation program and the luminosity function program are simply driven with a specific PERL interface, their outputs are directly displayed as an ASCII flux which can be saved in the user's hard disk and used by other programs without any difficulty.

The CMD generator is probably - at the moment- our more sophisticated WWW-based software. When it is invoked, a computation identification string of characters (CISC) is assigned. The files are handled and stored with this CISC as part of the file name -so even if multiple users from different parts of the world submit simulations at the same time, they can all be processed separately. When the computation is completed, an HTML page is created on-the-fly, displaying data output files and a pre-visualization made with GNUplot. The Perl module Net allows automatic e-mail sending for user notification.

Figure 7 summarizes the BaSTI Web-Tools general scheme. Some details have been omitted, like interaction with the plotting sofware GNUplot and random seeds download from http://www.random.org. At the moment all calculations are treated after each user's clicking, and consequently several computations can be running at the same time on our machine,

an occurrence that could seriously affect the computation time. Depending on the success of our on-line programs, we could implement as a new feature a more elaborated computations managing system that could "queue" the users requests, decreasing individual computation times.

\section{REFERENCES}

Bergeat, J., Knapik, A., \& Rutily, B. 2001, A\&A, 369, 178

Bessell, M. S. \& Brett, J. M. 1988, PASP, 100, 1134

Bono, G., Castellani, V., \& Marconi, M. 2000, ApJ, 529, 293

Cantiello, M., Raimondo, G., Brocato, E., \& Capaccioli, M. 2003, AJ, 125, 2783

Carpenter, J. M. 2001, AJ, 121, 2851

Cassisi, S., Salaris, M., Castelli, F., \& Pietrinferni, A. 2004, ApJ, 616, 498

Cassisi, S., Salaris, M., \& Irwin, A. W. 2003, ApJ, 588, 862

Di Criscienzo, M., Marconi, M., \& Caputo, F. 2004, ApJ, 612, 1092 
Dolphin, A. E. 2002, MNRAS, 332, 91

Dolphin, A. E., Saha, A., Skillman, E. D., Dohm-Palmer, R. C., Tolstoy, E., Cole, A. A., Gallagher, J. S., Hoessel, J. G., \& Mateo, M. 2003, AJ, 126, 187

Frogel, J. A. \& Cohen, J. G. 1982, ApJ, 253, 580

Gallart, C., Aparicio, A., Bertelli, G., \& Chiosi, C. 1996, AJ, 112, 2596

Gallart, C., Zoccali, M., \& Aparicio, A. 2005, ARA\&A, 43, 387

González, R. A., Liu, M. C., \& Bruzual A., G. 2004, ApJ, 611, 270

Grevesse, N. \& Noels, A. 1993, In: Origin and Evolution of the Elements, ed. V.-F. E. Prantzos N. \& C. M. (Cambridge University Press, Cambrigge), 14, (GN'93)

Harris, J. \& Zaritsky, D. 2004, AJ, 127, 1531

Holtzman, J. A., Gallagher, J. S., Cole, A. A., Mould, J. R., Grillmair, C. J., Ballester, G. E., Burrows, C. J., Clarke, J. T., Crisp, D., Evans, R. W., Griffiths, R. E., Hester, J. J., Hoessel, J. G., Scowen, P. A., Stapelfeldt, K. R., Trauger, J. T., \& Watson, A. M. 1999, AJ, 118, 2262

Iben, I. \& Truran, J. W. 1978, ApJ, 220, 980

James, F. 1990, Comput. Phys. Commun., 60, 329

Kroupa, P., Tout, C. A., \& Gilmore, G. 1993, MNRAS, 262, 545

Liu, M. C., Charlot, S., \& Graham, J. R. 2000, ApJ, 543, 644

Marconi, M., Caputo, F., Di Criscienzo, M., \& Castellani, M. 2003, ApJ, 596, 299

Marconi, M., Fiorentino, G., \& Caputo, F. 2004, A\&A, 417, 1101

Miller, B. W., Dolphin, A. E., Lee, M. G., Kim, S. C., \& Hodge, P. 2001, ApJ, 562, 713

Mollá, M., Ferrini, F., \& Gozzi, G. 2000, MNRAS, 316, 345

Moro, D. \& Munari, U. 2000, A\&AS, 147, 361

Mouhcine, M., González, R. A., \& Liu, M. C. 2005, MNRAS, 362, 1208

Pasian, F. 2004, Mem. Soc. Astr. It., 4, 86

Pietrinferni, A., Cassisi, S., Salaris, M., \& Castelli, F. 2004, ApJ, 612, 168 
-. 2006, ApJ, in press

Raimondo, G., Brocato, E., Cantiello, M., \& Capaccioli, M. 2005, AJ, 130, 2625

Reimers, D. 1975, Memoires of the Societe Royale des Sciences de Liege, 8, 369

Rocha-Pinto, H. J., Scalo, J., Maciel, W. J., \& Flynn, C. 2000, A\&A, 358, 869

Salaris, M. \& Weiss, A. 1998, A\&A, 335, 943

Salpeter, E. 1955, ApJ, 121, 161S

Searle, L., Wilkinson, A., \& Bagnuolo, W. G. 1980, ApJ, 239, 803

Skillman, E. D., Tolstoy, E., Cole, A. A., Dolphin, A. E., Saha, A., Gallagher, J. S., DohmPalmer, R. C., \& Mateo, M. 2003, ApJ, 596, 253

Vassiliadis, E. \& Wood, P. R. 1993, ApJ, 413, 641

Wagenhuber, J. 1996, Ph.D. Thesis

Wagenhuber, J. \& Groenewegen, M. A. T. 1998, A\&A, 340, 183

Westera, P., Lejeune, T., Buser, R., Cuisinier, F., \& Bruzual, G. 2002, A\&A, 381, 524

Woo, J.-H., Gallart, C., Demarque, P., Yi, S., \& Zoccali, M. 2003, AJ, 125, 754 
Table 1: Initial chemical composition of the model grid.

\begin{tabular}{|c|c|c|c|}
\hline$Z$ & $Y$ & {$[\mathrm{Fe} / \mathrm{H}]$ (scaled solar mixture) } & {$[\mathrm{Fe} / \mathrm{H}](\alpha$-enhanced mixture $)$} \\
\hline 0.0001 & 0.245 & -2.27 & -2.62 \\
\hline 0.0003 & 0.245 & -1.79 & -2.14 \\
\hline 0.0006 & 0.246 & -1.49 & -1.84 \\
\hline 0.0010 & 0.246 & -1.27 & -1.62 \\
\hline 0.0020 & 0.248 & -0.96 & -1.31 \\
\hline 0.0040 & 0.251 & -0.66 & -1.01 \\
\hline 0.0080 & 0.256 & -0.35 & -0.70 \\
\hline 0.0100 & 0.259 & -0.25 & -0.60 \\
\hline 0.0198 & 0.273 & +0.06 & -0.29 \\
\hline 0.0300 & 0.288 & +0.26 & -0.09 \\
\hline 0.0400 & 0.303 & +0.40 & +0.05 \\
\hline
\end{tabular}


Table 2: Summary of the BaSTI model and isochrone database.

\begin{tabular}{|c|c|c|c|c|c|c|c|c|}
\hline mixture & \multicolumn{3}{|c|}{ scaled-solar } & \multicolumn{4}{c|}{$\alpha$-enhanced } \\
\hline$\eta$ & \multicolumn{2}{|c|}{0.2} & \multicolumn{2}{c|}{0.4} & 0.2 & \multicolumn{2}{c|}{0.4} \\
\hline$\lambda_{O V}$ & 0 & 0.2 & 0 & 0.2 & 0 & 0.2 & 0 & 0.2 \\
\hline$N^{O}$ tracks & 20 & 20 & 40 & 20 & 20 & 20 & 40 & 20 \\
\hline$M_{\min }\left(\mathrm{M}_{\odot}\right)$ & 0.5 & 1.1 & 0.5 & 1.1 & 0.5 & 1.1 & 0.5 & 1.1 \\
\hline$M_{\max }\left(\mathrm{M}_{\odot}\right)$ & 2.4 & 2.4 & 10 & 10 & 2.4 & 2.4 & 10 & 10 \\
\hline$N^{O}$ isoc. & 63 & 44 & 54 & 44 & 63 & 44 & 54 & 44 \\
\hline Age $e_{\min }(\mathrm{Myr})$ & 30 & 30 & 30 & 30 & 30 & 30 & 30 & 30 \\
\hline Age $e_{\max }(\mathrm{Gyr})$ & 19 & 9.5 & 14.5 & 9.5 & 19 & 9.5 & 14.5 & 9.5 \\
\hline Color- $_{\text {eff }}$ & \multicolumn{6}{|c|}{$U B V R I J H L-$ ACS HST } \\
\hline
\end{tabular}


Table 3: Variable type index and sources used in the population synthesis program.

\begin{tabular}{|c|c|c|}
\hline Variables Type Index & Variable Type & Source for Instability Strip Boundaries \\
\hline 0 & no variable & \\
\hline 1 & fundamental RR Lyrae & Marconi et al. (2003); Di Criscienzo et al. (2004) \\
\hline 2 & first overtone RR Lyrae & Marconi et al. (2003); Di Criscienzo et al. (2004) \\
\hline 3 & fundamental anomalous Cepheid & Marconi et al. (2004) \\
\hline 4 & first overtone anomalous Cepheid & Marconi et al. (2004) \\
\hline 5 & fundamental classical Cepheid & Bono et al. (2000) \\
\hline
\end{tabular}


Table 4: Index for the isochrone sets employed by the SYNTHETIC MAN code.

\begin{tabular}{cl}
\hline Index & Isochrone set \\
\hline 1 & Scaled solar, no overshooting, $\eta=0.2$ \\
2 & Scaled solar, overshooting, $\eta=0.2$ \\
3 & Scaled solar, no overshooting, $\eta=0.4$ \\
4 & Scaled solar, overshooting, $\eta=0.4$ \\
5 & $\alpha$-enhanced, no overshooting, $\eta=0.2$ \\
6 & $\alpha$-enhanced, overshooting, $\eta=0.2$ \\
7 & $\alpha$-enhanced, no overshooting, $\eta=0.4$ \\
8 & $\alpha$-enhanced, overshooting, $\eta=0.4$ \\
\hline
\end{tabular}


Table 5: Available Star Formation Histories.

\begin{tabular}{ll}
\hline Stellar Population & Source \\
\hline NGC6822 (global SFH) & Gallart et al. (1996) \\
SMC (global SFH) & Harris \& Zaritskv (2004) \\
LMC (bar field) & Holtzman et al. (1999) \\
Local disk & Rocha-Pinto et al. (2000) \\
Milky Way bulge & Mollá et al. (2000) \\
Sextans A & Dolphin et al. (2003) \\
LGS3 & Miller et al. (2001) \\
\hline
\end{tabular}




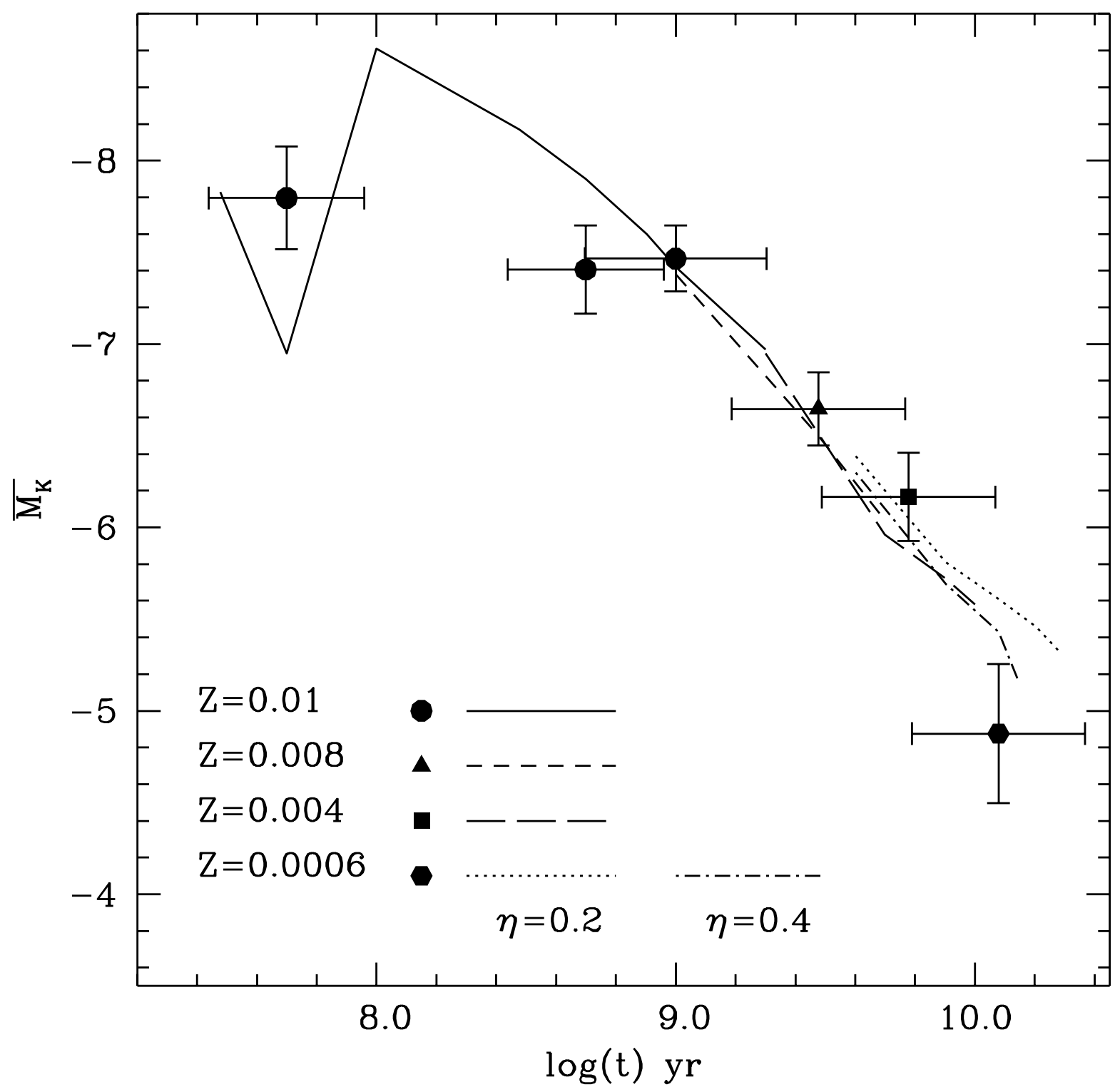

Fig. 1.- Run of the $K$-band SBF magnitudes of Magellanic Cloud superclusters as a function of their age. Theoretical SBF magnitudes for the appropriate metallicity and age range (denoted with different line styles) of the individual superclusters (superclusters of different metallicities are denoted with different symbols) are also displayed. These theoretical values are obtained from the scaled-solar isochrones including overshooting and with $\eta=0.2$, extended along the TP-AGB phase. At $Z=0.0006$ we display also values obtained with $\eta=0.4$ (dashed-dotted line - see text for details). 


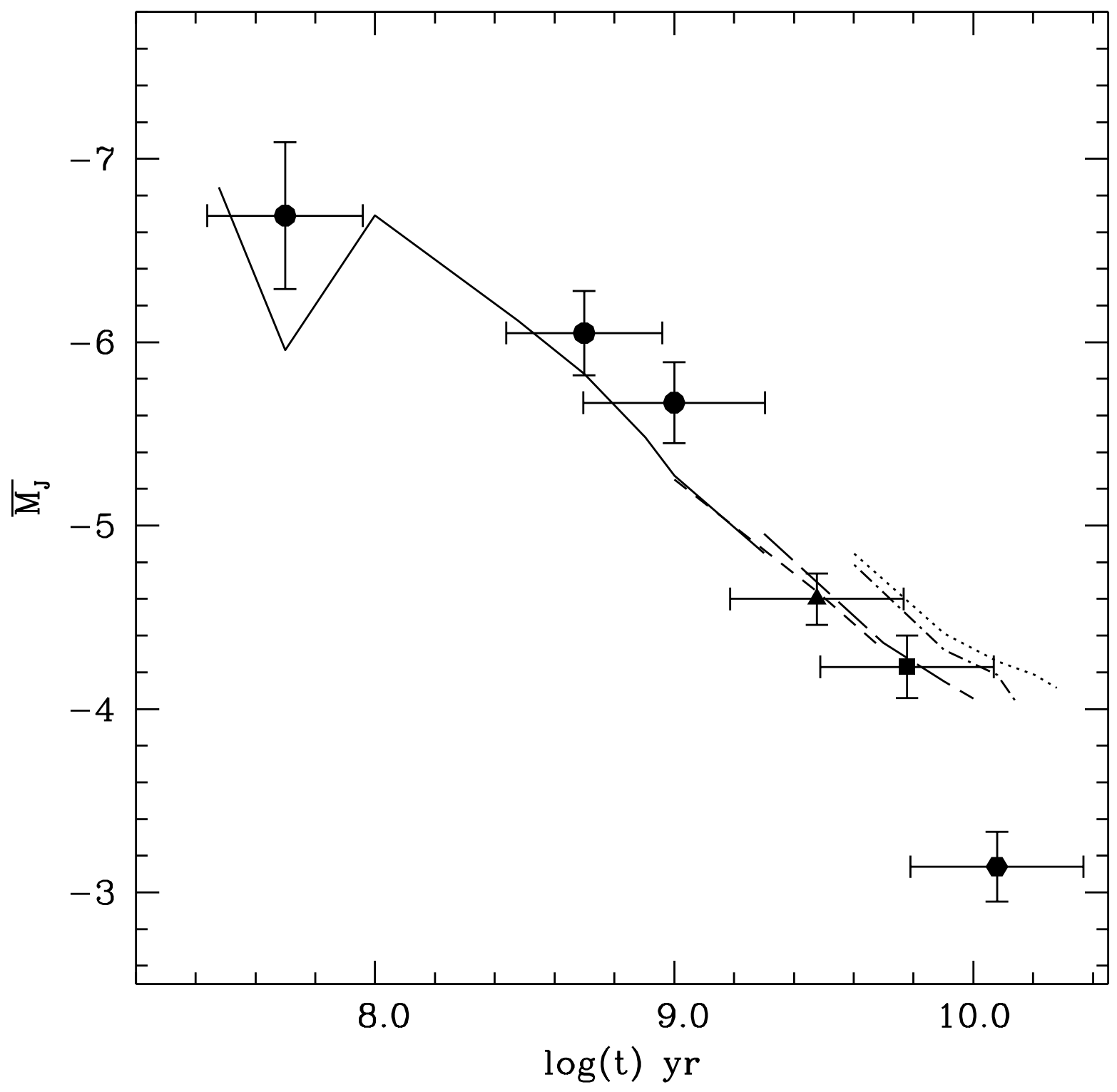

Fig. 2.- As in Fig. 1, but for the $J$-band SBF magnitudes (see text for details). 


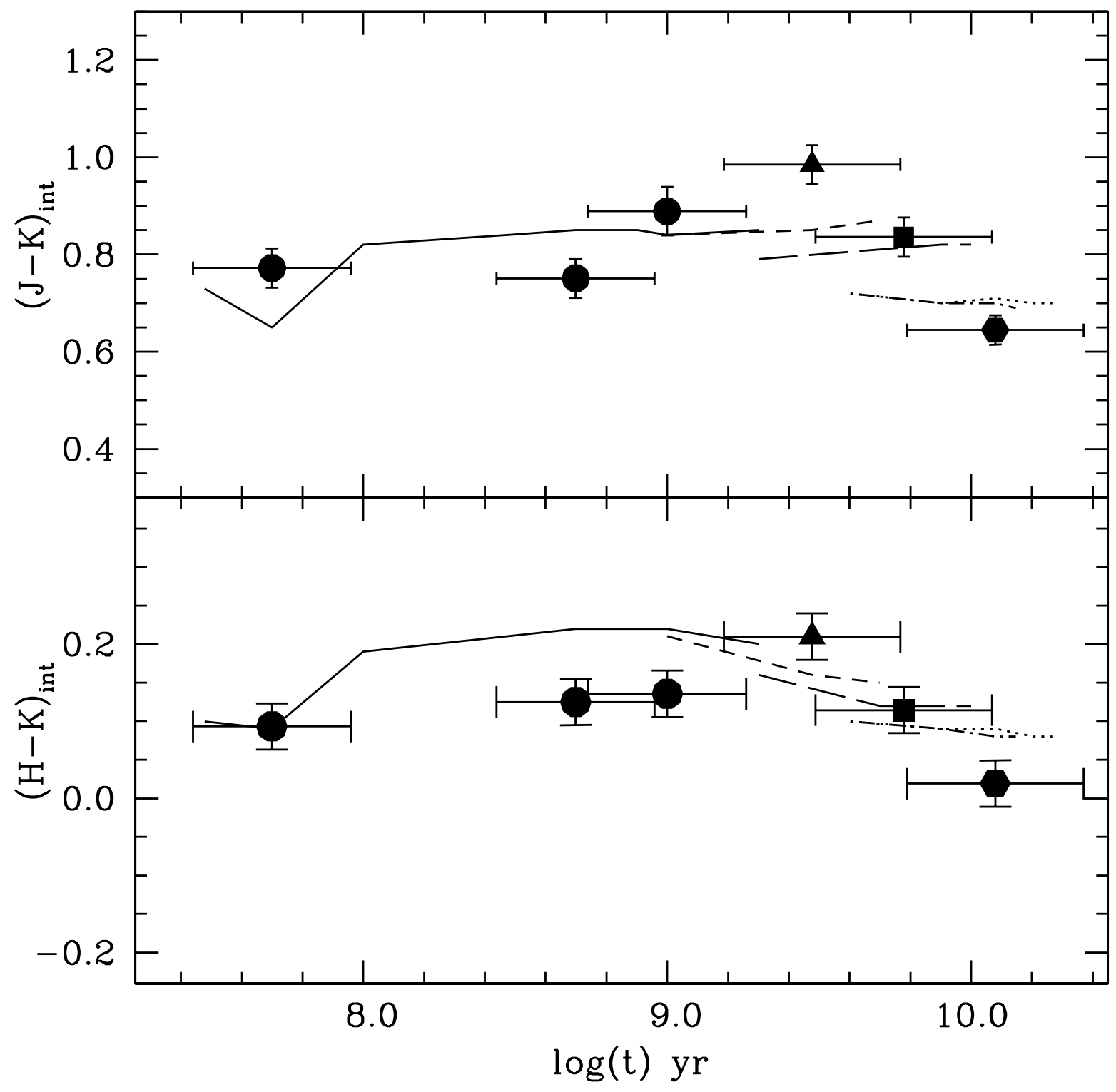

Fig. 3.- As in Fig. 1, but for the integrated $(H-K)$ and $(J-K)$ colors of Magellanic Cloud superclusters (see text for details). 


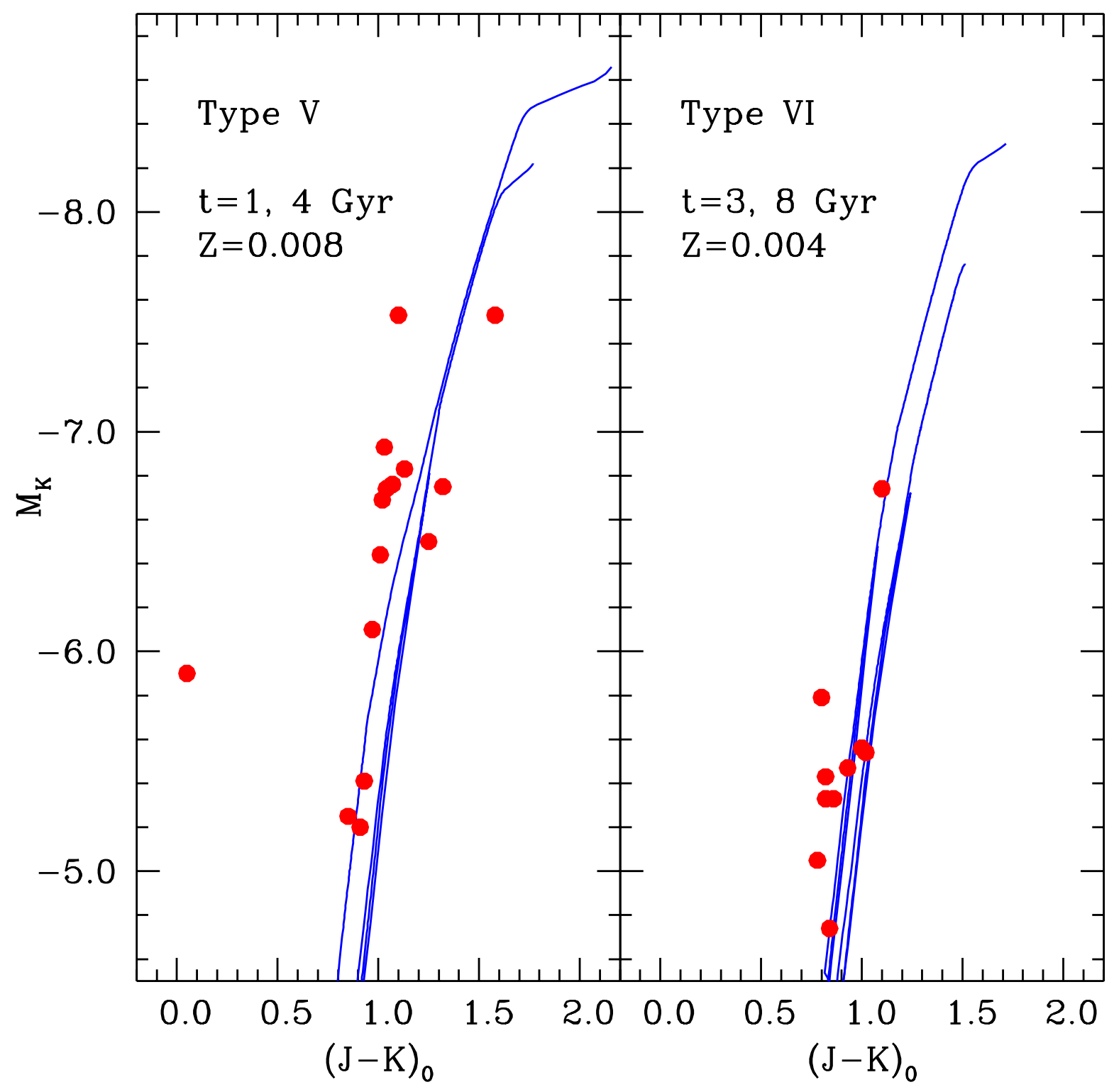

Fig. 4.- Cumulative $K-(J-K)$ CMDs of AGB stars in a sample of LMC clusters. Theoretical isochrones for the appropriate age and metallicity range are also displayed (see text for details). 


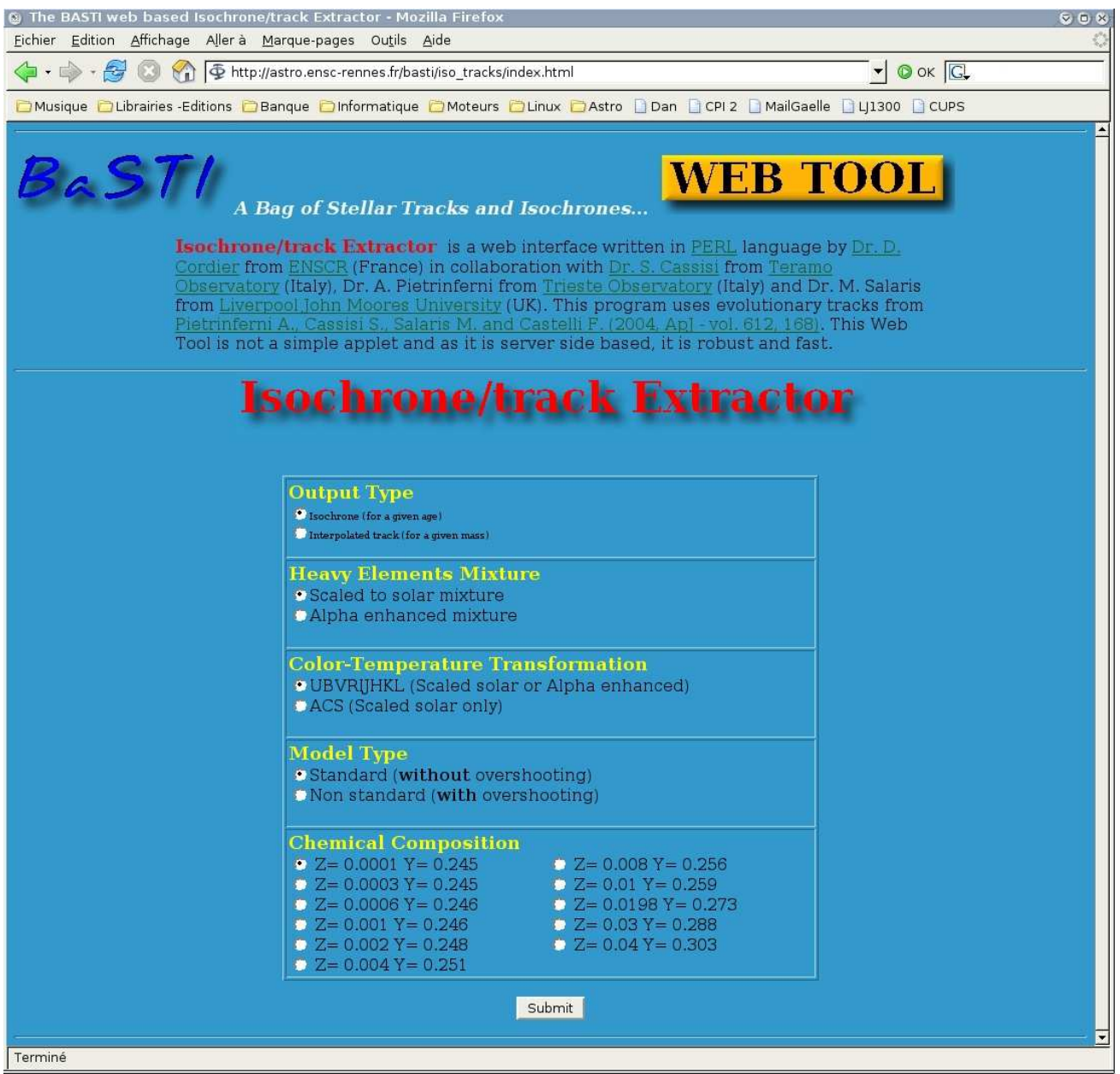

Fig. 5.- Web interface for the tool dedicated to isochrones and evolutionary tracks. 


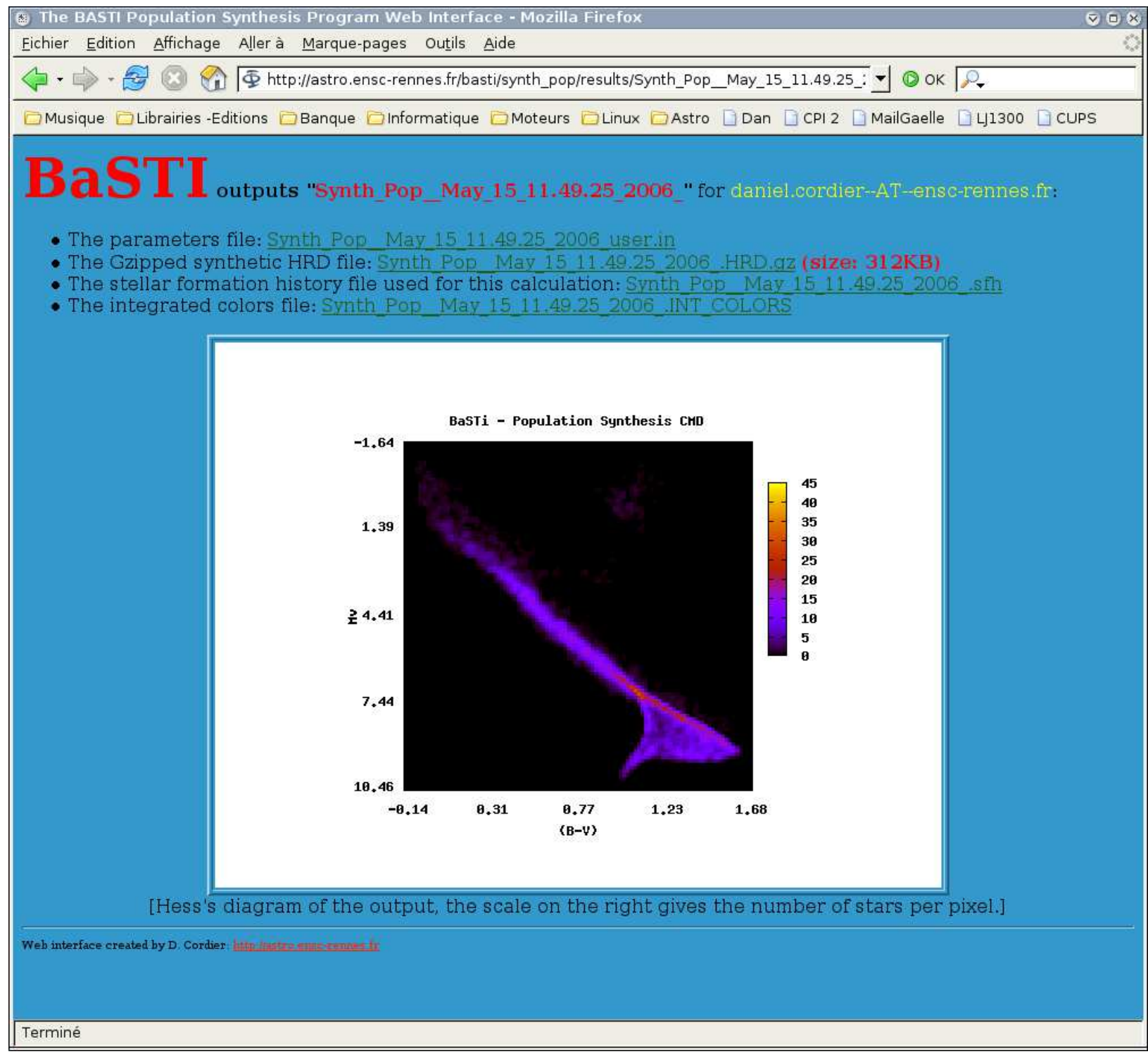

Fig. 6. - Example of the output web-page for the SYNTHETIC MAN web-tool. 


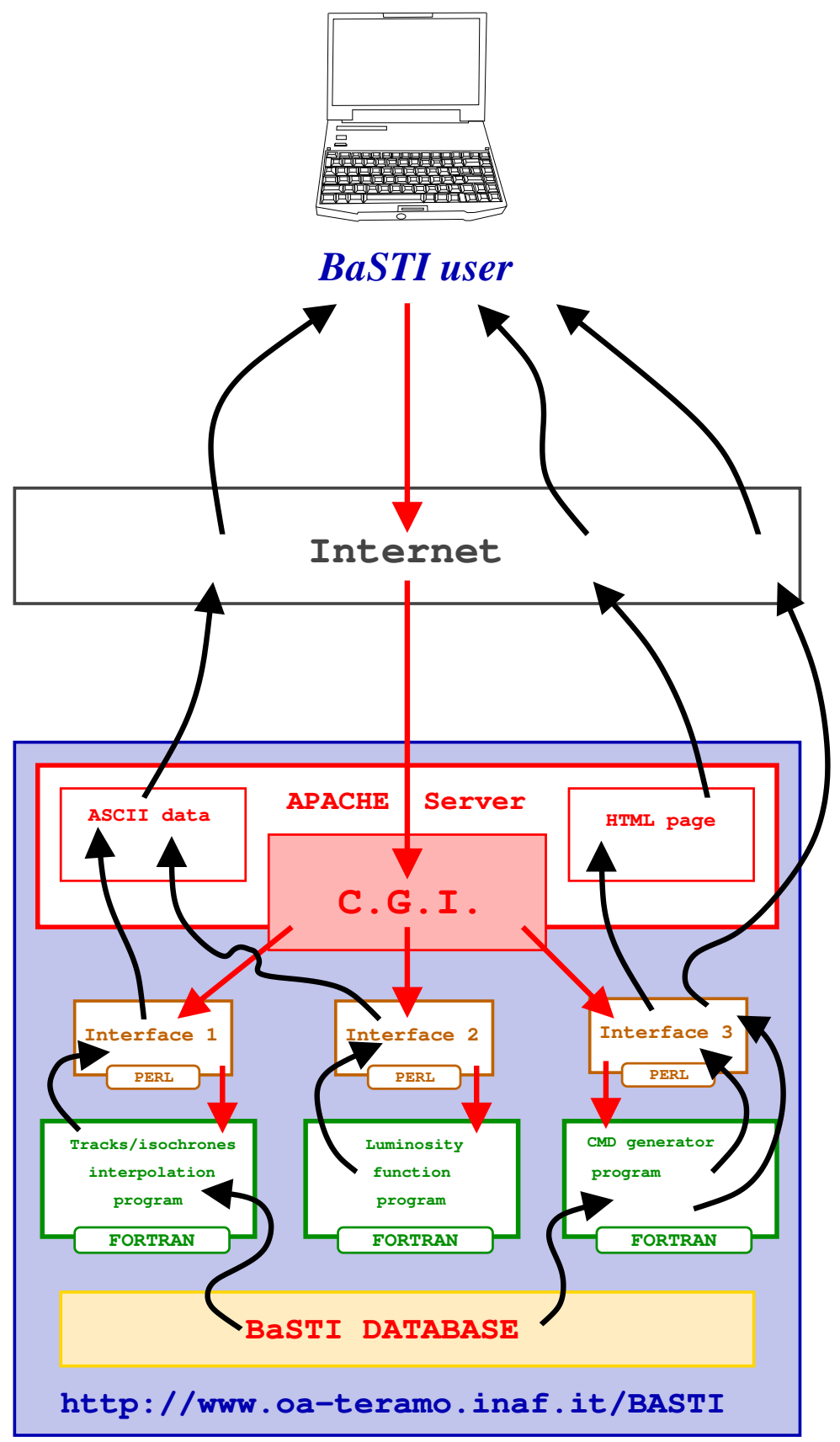

Fig. 7.- Data fluxes in the BaSTI web tools. Random number generator seeds provided by http://www.random.org has been omitted. 